\title{
Multiparticle entanglement as an emergent phenomenon
}

\author{
Nikolai Miklin, Tobias Moroder, and Otfried Gühne \\ Naturwissenschaftlich-Technische Fakultät, Universität Siegen, Walter-Flex-Str. 3, 57068 Siegen, Germany
}

(Dated: August 24, 2018)

\begin{abstract}
The question whether global entanglement of a multiparticle quantum system can be inferred from local properties is of great relevance for the theory of quantum correlations as well as for experimental implementations. We present a method to systematically find quantum states, for which the two- or three-body marginals do not contain any entanglement, nevertheless, the knowledge of these reduced states is sufficient to prove genuine multiparticle entanglement of the global state. With this, we show that the emergence of global entanglement from separable local quantum states occurs frequently and for an arbitrary number of particles. We discuss various extensions of the phenomenon and present examples where global entanglement can be proven from marginals, even if entanglement cannot be localized in the marginals with measurements on the other parties.
\end{abstract}

PACS numbers: 03.65.Ta, 03.65.Ud

\section{INTRODUCTION}

The relations between global properties of a system and the properties of its parts are central for many debates in science. In the field of physics these relations are often captured by the notion of complexity and, although a fully developed theory of complexity is still missing, many aspects have been discovered. An interesting concept in these discussions is the notion of emergence, meaning that at a global scale properties may be present, which are not present in the parts of the system. A central property of complex quantum systems is the possibility of being entangled, meaning in the simplest case that the wave function of the system does not factorize. Interestingly, it was already noted by Erwin Schrödinger in his first work on entanglement that this notion concerns also the relation between global and local properties 1. The study of this relation is known as the marginal problem or representability problem, and, although it has been studied for decades, it remains a key problem in quantum mechanics 2 .

With the advent of quantum information processing the question to which extent global entanglement properties can be inferred from local data can be reformulated in a precise mathematical manner and the tools of entanglement theory can be used to tackle it. If pure states are considered, then one can clearly infer entanglement from local properties since the reduced states of an entangled states are not pure anymore. Recently, this approach has been refined significantly by characterizing the single-particle reduced states that belong to certain classes of multiparticle entanglement [3, 4]. Nevertheless, if the purity of the global state is assumed the results cannot be used for any experimental implementation, since noise and decoherence always lead to mixed states. For multiparticle mixed states, it is clear that if the marginals contain already entanglement, then the global state must be entangled, too. Surprisingly, however, there are multiparticle quantum states where the two-particle marginals are not entangled at all, but nevertheless entanglement of the global state follows already
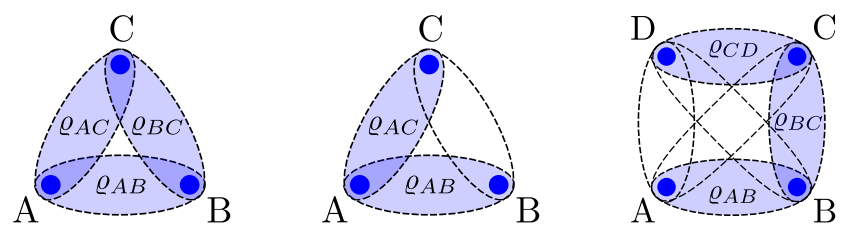

FIG. 1: Possible applications of method provided in this paper. Left: The method finds systematically three-particle states with no entanglement in the two-qubit marginals $\varrho_{A B}$, $\varrho_{B C}$, and $\varrho_{A C}$, where nevertheless global genuine multiparticle entanglement can be proven from knowing the separable marginals only. Middle, right: Possible variations of the problem considered in the paper. All two-body reduced states are required to be separable (shown by dashed ellipses), but only some (denoted by blue filled ellipses) are known and sufficient to prove that all particles are entangled.

from the marginals. This means that global entanglement can be proven by looking at separable marginals only and in this way, entanglement may appear as an emergent phenomenon. This has first been observed for entanglement in spin models and in the context of spin squeezing, where the task of proving entanglement from two-body marginals arises naturally [5, 6. The same observation has been made with the violation of Bell inequalities: here, the marginals may be compatible with a local hidden variable model, but such a model can be excluded for the global state by considering the marginals only [7, 8].

In all these works, however, only the simplest form of multiparticle entanglement was considered. Namely, it was asked whether or not the global state factorizes, or in technical terms, is fully separable. A state which does not factorize is entangled, but this does not mean that all particles are entangled. If all particles are entangled the state is called genuine multiparticle entangled and this property is much more difficult to infer from the marginals. In fact, there has been only one recent example of a three-qubit state, where the genuine multiparticle entanglement can be concluded from separable marginals 9]. However, this example and the underlying 
techniques are tailored to a specific situation and are not straightforward to generalize in any direction.

In this paper, we present a systematic method to find multiparticle quantum states where genuine multiparticle entanglement can be proven from separable marginals (see Fig. 1). Implementing this method numerically we find various examples for up to five particles. Based on these findings we provide a scheme for constructing of states with the desired properties for any number of particles, proving the universality of the described phenomenon. Furthermore, we consider possible extensions of the problem. Situations where only a subset of the separable two-body marginals is sufficient to prove entanglement, can easily be identified, as well as the case, where additionally the separability of higher-order marginals is given. Finally, a direct extension of our method delivers states where no entanglement can be generated between two arbitrary particles, even if measurements are made on the other particles. Still, global entanglement can be proven from the marginals. This shows that localizable entanglement is not a precondition for genuine multiparticle entanglement.

\section{STATEMENT OF THE PROBLEM}

Before explaining our method is it useful to recall the main definitions. First, a two-particle state $\varrho_{A B}$ is separable, if it can be written as a convex combination of product states, $\varrho_{A B}^{\mathrm{sep}}=\sum_{k} p_{k} \varrho_{A}^{k} \otimes \varrho_{B}^{k}$, where the $p_{k}$ form a probability distribution. Otherwise the state is entangled. If three or more particles are considered, different kinds of entanglement arise. To start, a three-particle state is fully separable, if it is of the form $\varrho_{A B C}^{\text {fsep }}=\sum_{k} p_{k} \varrho_{A}^{k} \otimes \varrho_{B}^{k} \otimes \varrho_{C}^{k}$, otherwise it contains some entanglement. But this does not mean that all particle are entangled, already the entanglement of two particles suffices to exclude full separability. The question whether all particles are entangled or not leads to the notion of genuine multiparticle entanglement and biseparability. A three-particle state is biseparable, if it is of the form

$$
\varrho_{A B C}^{\mathrm{bisep}}=p_{1} \varrho_{A \mid B C}^{\mathrm{sep}}+p_{2} \varrho_{B \mid A C}^{\mathrm{sep}}+p_{3} \varrho_{C \mid A B}^{\mathrm{sep}},
$$

where the state $\varrho_{A \mid B C}^{\mathrm{sep}}=\sum_{k} p_{k} \varrho_{A}^{k} \otimes \varrho_{B C}^{k}$ is separable for the partition $A \mid B C$ etc. If a state is not biseparable, it is genuine multiparticle entangled. Clearly, it is more difficult to prove that a state is genuine multiparticle entangled than proving that it just contains some entanglement, as more conditions have to be fulfilled.

Now we are able to define the problem in a precise manner. For three particles, we want to find a state $\varrho$, such that: (i) All two-body marginals (reduced states) are separable, and (ii) The state $\varrho$ itself is genuine multiparticle entangled and the entanglement can be proved from the two-body marginals.

In other words, the two conditions state that the separable marginals are only compatible with global genuine multipartite entanglement. It is important to note that in our problem uniqueness of the global state is not required and the only condition is that all global states, compatible with the marginals, must be genuine multiparticle entangled. One can extend the conditions in various ways. Concerning the first condition, one can require for four or more particles in addition to the two-body marginals that also the three-body marginals are fully separable. The second condition can be modified such that only some of the two-body marginals are known, while all of them are still separable (see Fig. 1). Clearly, both modifications make it more difficult to find the desired states and it is not clear, whether states with these properties exist at all. It is one of the main results of the present paper that for all reasonable modifications of the problem the corresponding states can be found.

\section{CONSTRUCTION METHOD}

Our method for constructing states with the desired properties is formulated as an iteration process consisting of two optimization problems, which are semidefinite programs (SDPs) and can thus be solved efficiently with freely available tools [10, 11. The algorithm is based on the approach to multiparticle entanglement with PPT mixtures [12]. This approach verifies whether or not a given state $\varrho$ is a mixture of states which have a positive partial transpose (PPT) for the different bipartitions [16]. So the test checks whether

$$
\varrho_{A B C}^{\mathrm{pptmix}}=p_{1} \varrho_{A \mid B C}^{\mathrm{ppt}}+p_{2} \varrho_{B \mid A C}^{\mathrm{ppt}}+p_{3} \varrho_{C \mid A B}^{\mathrm{ppt}} .
$$

Since separable states are also PPT, any state that fulfills the biseparability condition from Eq. (1) will also be a PPT mixture according to Eq. (2). Consequently a state that is not a PPT mixture must be genuine multiparticle entangled. The question whether a state is a PPT mixture can directly be decided via SDPs, most importantly, it can also be decided when only partial information on the state is available (see also below).

Now we describe the steps of the searching algorithm. We formulate it first for qubits, the extension to higherdimensional systems is explained afterwards.

Step 1. In the first part we apply the criterion for PPT mixtures to a random initial state $\varrho_{0}$ using only its marginals. For that, we have to solve the SDP

$$
\begin{aligned}
\underset{\mathcal{W}, P_{M}, Q_{M}}{\operatorname{minimize}} & \operatorname{tr}\left(\mathcal{W} \varrho_{0}\right), \\
\text { subject to: } & \operatorname{tr}(\mathcal{W})=1, \text { where } \\
& \mathcal{W}=\sum_{i j} w_{i j}^{\alpha, \beta} \sigma_{i}^{\alpha} \otimes \sigma_{j}^{\beta} \otimes \mathbb{1}^{\otimes(N-2)}+\text { perm. } \\
& \text { and for all bipartitions } M \mid \bar{M}: \\
& \mathcal{W}=P_{M}+Q_{M}^{T_{M}}, Q_{M} \geq 0, P_{M} \geq 0 .
\end{aligned}
$$

This optimization program means that for the state $\varrho_{0}$ we construct the optimal $\mathcal{W}$, which is a so-called decomposable witness for all bipartitions $M \mid \bar{M}$. If the expectation 
value $\operatorname{tr}\left(\mathcal{W} \varrho_{0}\right)$ for such a witness is negative, the state is genuine multipartite entangled, more details can be found in Ref. [12. The first constraint on the witness' trace maximizes the white-noise tolerance of the entanglement detection. The second linear constraint requires the witness to contain only two-body correlations, since we want to certify entanglement of our states from their two-body marginals only. Permutations are taken for all pairs $\alpha, \beta$ of qubits. This constraint is additional to the original PPT mixture problem and it is one of the algorithm's main features.

Step 2. In the second part of the iteration we determine the state that gives the most negative value for the given witness from the first step. In line with our approach, we require separability of the reduced two-party marginals. For the case of qubits this part can be formulated as a simple SDP, since a two-qubit state is separable, if and only if it is PPT. So we consider:

$$
\begin{array}{cl}
\underset{\varrho}{\operatorname{minimize}} & \operatorname{tr}(\mathcal{W} \varrho) \\
\text { subject to: } & \varrho \geq 0, \operatorname{tr}(\varrho)=1 \\
& \text { and for all } \alpha, \beta \quad \varrho_{\alpha \beta}^{T_{\alpha}} \geq 0
\end{array}
$$

where $\alpha, \beta$ denote pairs of qubits and $\varrho_{\alpha \beta}$ is the two-qubit marginal.

Combining both steps 1 and 2 and putting the output $\varrho$ of the second part as an input into the first part one can run an iteration process. If, at some point of the iteration the second step gives a negative value, we found already a state that has separable two-body marginals, where the entanglement can be proven from the marginals only. In practice, if a pure random state is taken as a seed of the algorithm, the iteration process typically gives a state with the desired properties after the second or the third iteration. Running the iteration further maximizes the entanglement and noise robustness of the state, while keeping the desired properties. Besides, the output states for different inputs $\varrho_{0}$ mainly differ only up to some local unitary transformation.

The algorithm can be extended to modifications of the problem in several ways. First, if higher-dimensional systems are considered, one has to use the appropriate generalizations of the Pauli matrices in the construction of the witness in Eq. (3). Also, in higher dimensions the PPT criterion is not necessary for separability, so for the state resulting in the second step separability of the marginals is not guaranteed. Nevertheless, if a state is found, the separability can later be checked with existing effective algorithms for proving separability of quantum states [13, 14. Second, if only some of the marginals are known [as in Fig. (1)] one just has to modify the definition of the witness in Eq. (3) and take only correlations from the known marginals. Finally, in the case of more than three parties, if also the full separability of the three-body marginals is required, one can modify the second step by changing the conditions that now the three-body marginals are PPT for any bipartition, and later verify the full separability of them with existing approaches [13, 14.

\section{RESULTS}

\section{A. Three qubits}

Although a state with the desired properties for three qubits is already known (see Ref. 9] and Appendix A), we start with this case, as this allows to explain our methods.

Taking the most robust algorithm's final state, applying local unitary operations and searching for an analytical expression, we find the following state which has separable two-body marginals which suffice to prove genuine multiparticle entanglement:

$$
\begin{aligned}
\varrho_{N}^{(3)} & =\frac{2}{3}|\xi\rangle\left\langle\xi\left|+\frac{1}{3}\right| \bar{W}_{3}\right\rangle\left\langle\bar{W}_{3}\right|, \\
|\xi\rangle & =\sqrt{\frac{1}{3}}\left|W_{3}^{*}\right\rangle+\sqrt{\frac{2}{3}}|111\rangle,
\end{aligned}
$$

where $\left|\bar{W}_{3}\right\rangle=(|011\rangle+|101\rangle+|110\rangle) / \sqrt{3}$ and $\left|W_{3}^{*}\right\rangle=$ $\left(e^{i \frac{\pi}{3}}|001\rangle+e^{-i \frac{\pi}{3}}|010\rangle-|100\rangle\right) / \sqrt{3}$ is a $W$-state with equally distributed phases. Note that besides these phases the state itself would be permutationally invariant. However, the asymmetry is necessary, since it can be shown that for symmetric states the studied phenomenon cannot exist [6] 17. We add that one can also see the set of marginals $\varrho_{A B}, \varrho_{B C}$ and $\varrho_{A C}$ as the output of the algorithm, we will discuss below the extent to which the marginals determine the state completely.

To compare our result with the result from Ref. [9], we note that the phenomenon described here is not fragile, as can be seen by the white noise tolerance. We consider the target state mixed with white noise $\varrho(p)=$ $(1-p) \varrho_{N}^{(3)}+p \mathbb{1} / 8$ and ask, for which values of $p$ the state has still the desired properties. Clearly, the marginals remain separable if white noise is added, so one only has to check whether the three-qubit entanglement can be proven from the marginals. One finds that the state in Eq. (5) remains this property with possible $13.7 \%$ of white-noise added, which means that this phenomenon is quite robust and it is realistic that this phenomenon can be observed experimentally. In addition, the white-noise tolerance of the state itself is $28.6 \%$, but then the entanglement cannot be proven from the marginals only. For comparison, the state from Ref. [9] keeps its properties only up to $5.2 \%$ of possible white-noise. Note that such estimates are impossible with the methods from Ref. 9, this it is another advantage of our algorithm.

\section{B. Four and five qubits}

For four qubits we find many analytical examples of states that have the desired properties. Remarkably, there are now also pure states with separable marginals, 


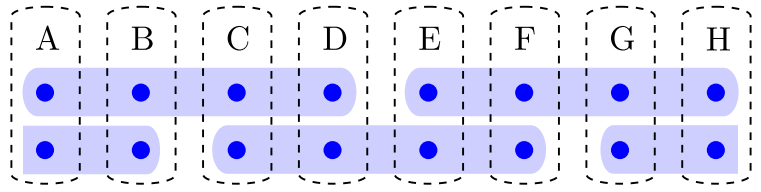

FIG. 2: Illustration of the construction of the desired state for eight parties. Dashed lines represent different parties, each party possesses two qubits. Blue rounded rectangles depict the entangled four-qubit state $\left|N^{(4)}\right\rangle$ from Eq. (6).

from which entanglement can be proved. One of the simplest solutions for four qubits is a Dicke-type state without one term and with one $\pi$-phase:

$\left|N^{(4)}\right\rangle=\frac{1}{\sqrt{5}}(|0011\rangle+|0101\rangle+|0110\rangle+|1001\rangle-|1010\rangle)$.

After local unitaries this state may also be expressed as a cluster state with an extra term $\left|N^{(4)}\right\rangle=2 / \sqrt{5}\left|C L_{4}\right\rangle+$ $1 / \sqrt{5}|0110\rangle$. This state has $21.2 \%$ white-noise tolerance (here and later we mean by white-noise tolerance the tolerance of the studied properties). A further property is that the state $\left|N^{(4)}\right\rangle$ is uniquely determined by its twobody marginals, this fact has far-reaching consequences as we will see below. There are other four-qubit states with the desired properties. The most entangled state, which we found for four-qubits is given in Appendix B and in Appendix $\mathrm{C}$ we provide a five-qubit example.

\section{Generalization to more particles}

Using the state in Eq. (6) one can construct examples of states for an arbitrary number of particles. We can formulate:

Observation. For any number of particles greater than four one can find a pure state with separable two-body marginals, where genuine multiparticle entanglement can be proven from these marginals only.

We illustrate the construction for eight parties, where each of the parties possesses a four-level system, represented by two qubits, so the total system consists of 16 qubits. We can distribute four copies of the state $\left|N^{(4)}\right\rangle$ in Eq. (6) as depicted in Fig. (2). Now every two-party marginal is separable since it is a direct product of two two-qubit separable states. Furthermore, knowledge of the two-party marginals implies knowledge on the states $\left|N^{(4)}\right\rangle$ and their distribution among the 16 qubits, since $\left|N^{(4)}\right\rangle$ is uniquely determined by its marginals. So the state of eight parties is uniquely determined by its twobody marginals as well. The global state is pure and does not factorize for any bipartition of the eight parties, so it is genuine multiparticle entangled and the entanglement can be proven from the separable marginals.

A similar reasoning is valid for any number of parties. For five parties one takes two copies of the state $\left|N^{(4)}\right\rangle$ and distributes the first copy to the parties $A, B, C, D$ and the second copy to the parties $B, C, D, E$, for other numbers of qubits the construction is analogous. Note that the purity of the state $\left|N^{(4)}\right\rangle$ is essential for the argument, so the construction would not work with other states, e.g. the state from Ref. [9]. Also, we needed that the state is uniquely determined by its two-qubit reduced states. Below we discuss this property for the other states we found using the method.

\section{Uniqueness of the global state}

The property of uniqueness of the global sate was noted before for the state from Ref. 9. In fact, this is the case for both three and four-qubit states from the Eqs. (5, 6). However, this property does not necessarily follow from the constraints and a counterexample for four qubits is the following state:

$$
|\Psi\rangle=\frac{1}{\sqrt{2}}\left|\widetilde{D_{2}^{4}}\right\rangle+\frac{1}{\sqrt{2}}\left|G H Z_{4}\right\rangle
$$

with

$$
\begin{array}{r}
\left|G H Z_{4}\right\rangle=\frac{1}{\sqrt{2}}(|0000\rangle+|1111\rangle), \\
\left|\widetilde{D_{2}^{4}}\right\rangle=\frac{1}{\sqrt{6}}(|0011\rangle+|0101\rangle+|0110\rangle \\
\left.+e^{i \varphi}|1001\rangle+e^{i \varphi}|1010\rangle+e^{-i \varphi}|1100\rangle\right),
\end{array}
$$

and $\varphi=\arccos (-1 / 3)$.

In this case, the two-qubit marginals are also compatible with the state of the same form as Eq. (7), but with the opposite phases $\varphi \rightarrow-\varphi$, meaning that there is a set of states, which are convex combinations of $|\Psi(\varphi)\rangle$ and $|\Psi(-\varphi)\rangle$ and which are compatible with the same reduced two-qubit states. This demonstrates that in our problem the set of two-body reduced states need not be compatible with only one global state, the only condition is that set of global states, compatible with these marginals, must be enclosed into the set of genuine multiparticle entangled states.

\section{EXTENSIONS OF THE PROBLEM}

First, one may ask whether there is any four-qubit state with separable two and three-body marginals, where the genuine multiparticle entanglement can be proven from the two-qubit reduced states. Our approach can also be used to find such states, details are given in Appendix D Second, there may be the possibility to detect entanglement from only a part of two-body correlations (see Fig. 1). For the three-qubit configuration where only the correlations between Alice and Bob and Alice and Charlie are known, we present a state in Appendix E. For the four-qubit case we note that the genuine multiparticle entanglement of the state from the 


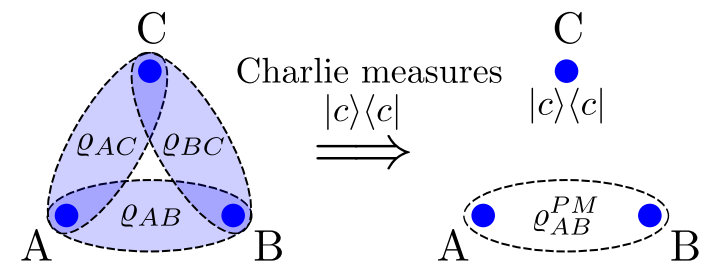

FIG. 3: Illustration of the emergence of multiparticle entanglement in the case of vanishing localizable entanglement. The post-measurement state $\varrho_{A B}^{P M} \sim\left\langle c\left|\varrho_{A B C}\right| c\right\rangle$ is separable after arbitrary measurements of Charlie, but knowing the reduced states $\varrho_{A B}, \varrho_{A C}$, and $\varrho_{B C}$ is still enough to prove genuine tripartite entanglement of the original state $\varrho_{A B C}$.

Eq. (7) can be detected by knowing only $\varrho_{A B}, \varrho_{B C}$, and $\varrho_{C D}$ with possible $3 \%$ of white noise added. As a further extension, we found also examples for three threedimensional systems, see Appendix F for details. Judging by the simulations we made, it seems that it is possible to find a state for any imaginable configuration of measurable correlations as soon as they contain correlations between every bipartition of the system.

Finally, we demonstrate that an extension of our method can be used to find a three-qubit state, where the marginals do not contain any entanglement, even after arbitrary measurements on the third particle. Nevertheless, global entanglement can be proved from the marginals only.

To start, one can ask whether the two-particle marginals are also separable, if the third party has made a measurement, described by a projector $|c\rangle\langle c|$ (see Fig. 3). For projective measurements, the postmeasurement state is described by $\varrho_{A B}^{P M} \sim\left\langle c\left|\varrho_{A B C}\right| c\right\rangle$ and one can see that if this state is separable for all $|c\rangle$ then the marginal is also separable, if Charlie makes a generalized measurement or an operation [18. So the question arises whether there exist genuine tripartite entangled states with the property that whatever projective measurement one party performs, the remaining two parties share a separable state. One can think about it as a state with no creatable entanglement in its subsystems, or a state with vanishing localizable entanglement [15. In addition, we want the entanglement to be provable from the two-body marginals $\varrho_{i j}$, but the knowledge of the conditional states $\varrho_{i j}^{P M}$ should not be required for the entanglement proof.

To look for such a state one should add an infinite number of additional constraints to the semidefinite program, corresponding to all possible local projective measurements. This is, of course, infeasible. However, it is sufficient to add a finite number of constraints corresponding to some measurements (preferably equally distributed over the Bloch sphere) and require a strict positivity (e.g., $\left[\varrho_{A B}^{P M}\right]^{T_{A}} \geq \varepsilon \mathbb{1}$ for some $\epsilon>0$ ) of the partial transposition of the post-measurement states. If a state is finally found, one can check by direct numerical evaluation whether the post-measurement states are separable even for arbitrary measurements.

Applying this in practice, one directly finds states with the desired properties, an example is given in detail in Appendix G. This proves that genuine multiparticle entanglement can emerge, even if the localizable entanglement vanishes.

\section{CONCLUSIONS}

In conclusion, we have provided a method to study systematically the emergence of genuine multiparticle entanglement from separable marginals. Our findings show the rich structure of multiparticle entanglement, where essentially all possible entanglement properties of the marginals can be combined. We believe that the entanglement properties of the resulting quantum states deserve further study, moreover, it would be interesting to observe the here described effects experimentally.

\section{Acknowledgments}

We thank O. Gittsovich, C. Schwemmer, J. Siewert, C. Spee, G. Tóth, R. Uola and an anonymous referee for comments and discussions. This work has been supported by the DAAD, the EU (Marie Curie CIG 293993/ENFOQI), the ERC (Consolidator Grant 683107/TempoQ) the FQXi Fund (Silicon Valley Community Foundation) and the DFG.

\section{Appendix}

\section{A. The state found in Ref. [9]}

In Ref. [9] the authors provide an example of two-qubit marginal triplet, which is compatible with only one global state, which is genuine tripartite entangled. It is given by:

$$
\begin{aligned}
\varrho & =\frac{2}{3}|\xi\rangle\left\langle\xi\left|+\frac{1}{3}\right| 111\right\rangle\langle 111|, \\
|\xi\rangle & =\frac{1}{2}|010\rangle+\frac{1}{2}|100\rangle+\frac{1}{\sqrt{2}}|001\rangle .
\end{aligned}
$$

\section{B. The four-qubit state with the highest noise robustness}

The most noise-robust four-qubit state that we found contains a Dicke part and a GHZ part with the Dicke 
part having asymmetric amplitudes. It is given by:

$$
\begin{aligned}
|\Psi\rangle & \left.=\frac{1}{\sqrt{2}}|\eta\rangle+\frac{1}{\sqrt{2}} \mid \text { GH }_{4}\right\rangle, \\
|\eta\rangle & =\frac{1}{2 \sqrt{2}}(-i|0011\rangle+|0101\rangle+|0110\rangle \\
& -\sqrt{3}|1001\rangle+i|1010\rangle+|1100\rangle) .
\end{aligned}
$$

This state has white-noise tolerance of $22.4 \%$ and it is the closest one to the numerical solution.

\section{A five-qubit example}

The following state is a genuine multiparticle entangled five-qubit state with separable marginals, which are compatible with this state only.

$$
\begin{aligned}
& |\Psi\rangle=\frac{1}{\sqrt{6}}|00\rangle \otimes|000\rangle \\
+ & \frac{1}{\sqrt{8}}|11\rangle \otimes\left(\sqrt{\frac{2}{3}}|000\rangle-|001\rangle-|010\rangle-|100\rangle\right) \\
+ & \frac{1}{\sqrt{24}}|01\rangle \otimes\left(-|001\rangle+e^{-i \frac{\pi}{3}}|010\rangle+e^{i \frac{\pi}{3}}|100\rangle\right) \\
+ & \frac{1}{\sqrt{48}}|01\rangle \otimes\left(e^{-i \frac{2 \pi}{3}}|011\rangle+e^{i \frac{2 \pi}{3}}|101\rangle+|110\rangle\right) \\
+ & \frac{1}{\sqrt{24}}|10\rangle \otimes\left(-|001\rangle+e^{i \frac{\pi}{3}}|010\rangle+e^{-i \frac{\pi}{3}}|100\rangle\right) \\
+ & \frac{1}{\sqrt{48}}|10\rangle \otimes\left(e^{-i \frac{\pi}{3}}|011\rangle+e^{i \frac{\pi}{3}}|101\rangle-|110\rangle\right) .
\end{aligned}
$$

This state has $17.3 \%$ white-noise tolerance.

\section{A four-qubit state with separable two and three-body marginals}

To find the following example, we have used our method to find a state, where the two- and three-body marginals are all PPT, and still the entanglement can be proven from the two-body marginals. Our method results in the following state

$$
\begin{aligned}
\varrho_{N}^{(4)} & =\frac{1}{2}\left|\zeta_{1}\right\rangle\left\langle\zeta_{1}\left|+\frac{1}{2}\right| \zeta_{2}\right\rangle\left\langle\zeta_{2}\right| \\
\left|\zeta_{1}\right\rangle & =\sqrt{\frac{4}{5}}\left|G H Z_{4}\right\rangle+\sqrt{\frac{1}{5}}\left|\Psi^{+}\right\rangle_{A B} \otimes\left|\Psi^{+}\right\rangle_{C D}, \\
\left|\zeta_{2}\right\rangle & =\sqrt{\frac{2}{5}}(|0011\rangle+|1100\rangle)+\sqrt{\frac{1}{5}}\left|\Psi^{-}\right\rangle_{A B} \otimes\left|\Psi^{-}\right\rangle_{C D},
\end{aligned}
$$

where $\left|\Psi^{ \pm}\right\rangle=(|01\rangle \pm|10\rangle) / \sqrt{2}$ denote the Bell states. This state has the described properties, even if up to $21.8 \%$ of white-noise are added. However, while the PPT property of the two-body marginals implies separability this is not the case for the three-body marginals: Even if a three-qubit state is PPT for all bipartitions, this does not mean that it is fully separable. Therefore, we checked whether the three-qubit marginals are fully separable with the separability testing algorithm from Ref. [13] and found that if more than $13.5 \%$ of white noise is added, the marginals are indeed fully separable. This proves that the state $\varrho(p)=(1-p) \varrho_{N}^{(4)}+p \mathbb{1} / 16$ for $p \in[0.135,0.218]$ has the desired properties.

\section{E. Three-qubit case, where not all marginals are known}

A genuine multiparticle entangled state with all twobody marginals being separable and which can be detected from the correlations between $\mathrm{A}$ and $\mathrm{B}$ and $\mathrm{A}$ and $\mathrm{C}$ only (as depicted in Fig. 1) is given by:

$$
\begin{aligned}
\varrho & =\frac{1}{2}\left|\xi_{1}\right\rangle\left\langle\xi_{1}\left|+\frac{1}{2}\right| \xi_{2}\right\rangle\left\langle\xi_{2}\right| \\
\left|\xi_{1}\right\rangle & =\sqrt{\frac{1}{10}}\left(\sqrt{5}|000\rangle+\sqrt{4} e^{-i \frac{3}{4} \pi}|011\rangle+e^{-i \frac{3}{4} \pi}|101\rangle\right) \\
\left|\xi_{2}\right\rangle & =\sqrt{\frac{1}{10}}\left\{\sqrt{3}\left(|001\rangle+e^{i \frac{2}{3} \pi}|010\rangle+e^{-i \frac{1}{3} \pi}|100\rangle\right)+|111\rangle\right\} .
\end{aligned}
$$

This effect has $5 \%$ of white-noise tolerance.

\section{F. A three-qutrit example}

When considering higher dimensions, it is worth noting that for every example of states, which we present in this paper, there exist trivial extensions to higher dimensions. For instance, if we take a three-qubit state $\varrho_{N}^{(3)}$ from Eq. (5), then, as noted in Ref. [9], a family of states $\varrho_{d}^{(3)}=p_{1} \varrho_{N}^{(3)}+\sum_{m=2}^{d} p_{m}|m m m\rangle\langle m m m|\left(p_{1}>0, p_{m} \geq 0\right)$ satisfy all the conditions of the desired states. However, this extension is rather trivial and does not give the best possible effect for qudit states. Using our algorithm for the three-qutrit case, we have found the following state.

$$
\begin{aligned}
\varrho & =\frac{1}{2}\left|\eta_{1}\right\rangle\left\langle\eta_{1}\left|+\frac{1}{2}\right| \eta_{2}\right\rangle\left\langle\eta_{2}\right|, \\
\left|\eta_{1}\right\rangle & =\frac{1}{\sqrt{12}}(|000\rangle-|222\rangle)-\frac{i \sqrt{5}}{6}(|012\rangle+|021\rangle \\
& -|102\rangle+|120\rangle+|201\rangle+|210\rangle), \\
\left|\eta_{2}\right\rangle & =\frac{1}{\sqrt{6}}|111\rangle-\frac{\sqrt{5}}{6}(|012\rangle-|021\rangle+|102\rangle \\
& +|120\rangle+|201\rangle-|210\rangle) .
\end{aligned}
$$

for which the two-body marginals are PPT, but genuine multiparticle entanglement can be proven from them. The white-noise tolerance of the discussed properties of the state is $29.5 \%$. Separability of its reduced states can be proven using the algorithm [13] if $5.3 \%$ of white-noise is added. So, the three-qutrit state $\sigma=(1-p) \varrho+p \mathbb{1} / 27$ has for $p \in[0.053,0.275]$ the property that genuine multiparticle entanglement can be proven from separable marginals. 


\section{G. A three-qubit state without entanglement in the marginals after measurements}

The following state is a three-qubit state with separable marginals after an arbitrary projective measurement on one of the qubits but where the two-body correlations are sufficient to determine genuine multiparticle entanglement of the global state.

$$
\begin{aligned}
\varrho & =\frac{1}{3}\left|\chi_{1}\right\rangle\left\langle\chi_{1}\left|+\frac{1}{3}\right| \chi_{2}\right\rangle\left\langle\chi_{2}\right| \\
& +\frac{1}{6}\left|\chi_{3}\right\rangle\left\langle\chi_{3}\left|+\frac{1}{6}\right| \chi_{4}\right\rangle\left\langle\chi_{4}\right|,
\end{aligned}
$$

where the eigenvectors are

$$
\begin{aligned}
\left|\chi_{1}\right\rangle & =\sqrt{\frac{5}{21}}\left(|001\rangle+e^{-i \frac{\pi}{6}}|010\rangle+e^{-i \frac{3}{4} \pi}|100\rangle\right) \\
& +\sqrt{\frac{2}{21}}\left(e^{i \frac{\pi}{5}}|011\rangle+e^{i \pi}|101\rangle+e^{i \frac{\pi}{9}}|110\rangle\right), \\
\left|\chi_{2}\right\rangle & =\frac{1}{3} e^{i \frac{4}{5} \pi}|000\rangle+\sqrt{\frac{3}{7}}|111\rangle \\
& +\sqrt{\frac{1}{42}}\left(e^{i \frac{5}{6} \pi}|001\rangle+e^{-i \frac{2}{3} \pi}|010\rangle+e^{-i \frac{3}{5} \pi}|100\rangle\right) \\
& +\sqrt{\frac{7}{54}}\left(e^{-i \frac{3}{5} \pi}|011\rangle+e^{-i \frac{5}{9} \pi}|101\rangle+|110\rangle\right), \\
\left|\chi_{3}\right\rangle & =\frac{\sqrt{18}}{5}|000\rangle+\frac{1}{5} e^{i \frac{\pi}{5}}|111\rangle \\
& +\frac{\sqrt{2}}{5}\left(e^{i \pi}|001\rangle+e^{-i \frac{\pi}{2}}|010\rangle+e^{-i \frac{2}{5} \pi}|100\rangle\right), \\
\left|\chi_{4}\right\rangle & =\frac{1}{\sqrt{3}}\left(|001\rangle+e^{-i \frac{5}{6} \pi}|010\rangle+|100\rangle\right) .
\end{aligned}
$$

[1] E. Schrödinger, Die Naturwissenschaften 23, 807-812, 823-828, 844-849 (1935).

[2] A. J. Coleman, Rev. Mod. Phys. 35, 668 (1963).

[3] M. Walter, B. Doran, D. Gross, and M. Christandl, Science 340, 1205 (2013).

[4] A. Sawicki, M. Walter, and M. Kus, J. Phys. A 46, 055304 (2013).

[5] G. Tóth, Phys. Rev. A 71, 010301(R) (2005).

[6] G. Tóth, C. Knapp and O. Gühne, H. J. Briegel, Phys. Rev. A 79, 042334 (2009).

[7] L. E. Würflinger, J-D. Bancal, A. Acín, N. Gisin and T. Vértesi, Phys. Rev. A 86, 032117 (2012).

[8] J. Tura, R. Augusiak, A. B. Sainz, T. Vértesi, M. Lewenstein, and A. Acín, Science 344, 1256 (2014).

[9] L. Chen, O. Gittsovich, K. Modi and M. Piani, Phys. Rev. A 90, 042314 (2014).

[10] J. Löfberg, Yalmip : A toolbox for modeling and optimization in MATLAB, Proceedings of the CACSD Conference, Taipei, Taiwan, (2004).

[11] K. C. Toh, M. J. Todd, and R. H. Tutuncu, Optimization Methods and Software 11, 545 (1999).

[12] B. Jungnitsch, T. Moroder and O. Gühne, Phys. Rev.
This state keeps its properties with possible level of white noise of $2 \%$. As discussed in the corresponding section, to find this state we added a finite number of constraints, corresponding to various projective measurements, and then required strict positivity of the post-measurement states. In our implementation we defined $\sim 1000$ constraints and required the eigenvalues of the partial transposition of the post-measurement states to be larger than $\sim 10^{-4}$.
Lett. 106, 190502 (2011).

[13] J. T. Barreiro, P. Schindler, O. Gühne, T. Monz, M. Chwalla, C. F. Roos, M. Hennrich, and R. Blatt, Nature Physics 6, 943 (2010).

[14] H. Kampermann, O. Gühne, C. Wilmott, and D. Bruß, Phys. Rev. A 86, 032307 (2012).

[15] M. Popp, F. Verstraete, M. A. Martín-Delgado, and J. I. Cirac, Phys. Rev. A 71, 042306 (2005).

[16] The partial transpose of a state $\varrho=\sum_{i j, k l} \varrho_{i j, k l}|i\rangle\langle j| \otimes$ $|k\rangle\langle l|$ is given by $\varrho^{T_{A}}=\sum_{i j, k l} \varrho_{i j, k l}|j\rangle\langle i|\otimes| k\rangle\langle l|$ and for a separable state the partial transpose is positive semidefinite.

[17] Nevertheless, we found with our algorithm permutationally invariant states with the desired properties.

[18] The most general marginal after an operation on $C$ is given by $\varrho_{A B}^{P M}=\operatorname{Tr}_{C}\left[\mathbb{I}_{A} \otimes \mathbb{I}_{B} \otimes \Lambda_{C}\left(\varrho_{A B C}\right)\right]$, where $\Lambda_{C}(\rho)=\sum_{k} A_{k} \rho A_{k}^{\dagger}$ with $\sum_{k} A_{k}^{\dagger} A_{k} \leq \mathbb{1}$ is a tracenon-increasing operation. Using the polar decomposition $A_{k}=U\left[s_{1}|c\rangle\left\langle c\left|+s_{2}\right| c^{\perp}\right\rangle\left\langle c^{\perp}\right|\right]$ with $s_{i} \geq 0$ we have for a single term that $\operatorname{Tr}_{C}\left[A_{k}\left(\varrho_{A B C}\right) A_{k}^{\dagger}\right]=s_{1}\left\langle c\left|\varrho_{A B C}\right| c\right\rangle+$ $s_{2}\left\langle c^{\perp}\left|\varrho_{A B C}\right| c^{\perp}\right\rangle$ is separable as a convex combination of 
separable states, so also $\varrho_{A B}^{P M}$ is separable. 